\title{
Using Ultrasound and Inflammation to Improve Prediction of Ischemic Stroke: A Secondary Analysis of the Multi-Ethnic Study of Atherosclerosis
}

\author{
Hediyeh Baradaran $^{\mathrm{a}} \quad$ Alen Delic $^{\mathrm{b}} \quad \mathrm{Ka}-\mathrm{Ho}$ Wong ${ }^{\mathrm{c}}$ Nazanin Sheibanic \\ Matthew Alexander ${ }^{\mathrm{a}} \quad$ J. Scott McNally ${ }^{\mathrm{a}}$ Jennifer J. Majersik ${ }^{c}$ Adam De Havenon ${ }^{\mathrm{c}}$ \\ ${ }^{a}$ Department of Radiology, University of Utah, Salt Lake City, UT, USA; ${ }^{b}$ Department of Biostatistics, University of Utah, \\ Salt Lake City, UT, USA; ' Department of Neurology, University of Utah, Salt Lake City, UT, USA
}

\section{Keywords}

Cerebrovascular disease/stroke · Carotid ultrasound .

Atherosclerosis

\begin{abstract}
Introduction: Current ischemic stroke risk prediction is primarily based on clinical factors, rather than imaging or laboratory markers. We examined the relationship between baseline ultrasound and inflammation measurements and subsequent primary ischemic stroke risk. Methods: In this secondary analysis of the Multi-Ethnic Study of Atherosclerosis (MESA), the primary outcome is the incident ischemic stroke during follow-up. The predictor variables are 9 carotid ultrasound-derived measurements and 6 serum inflammation measurements from the baseline study visit. We fit Cox regression models to the outcome of ischemic stroke. The baseline model included patient age, hypertension, diabetes, total cholesterol, smoking, and systolic blood pressure. Goodness-of-fit statistics were assessed to compare the baseline model to a model with ultrasound and inflammation predictor variables that remained significant when added to the baseline model. Results: We included 5,918 participants. The primary outcome of ischemic stroke was seen
\end{abstract}

karger@karger.com www.karger.com/cee

Karger $\stackrel{\text { ' }}{5}$

GOPEN ACCESS
(C) 2021 The Author(s)

Published by S. Karger AG, Basel

This article is licensed under the Creative Commons AttributionNonCommercial-NoDerivatives 4.0 International License (CC BYNC-ND) (http://www.karger.com/Services/OpenAccessLicense) Usage and distribution for commercial purposes as well as any distribution of modified material requires written permission. in 105 patients with a mean follow-up time of 7.7 years. In the Cox models, we found that carotid distensibility (CD), carotid stenosis (CS), and serum interleukin-6 (IL-6) were associated with incident stroke. Adding tertiles of CD, IL-6, and categories of CS to a baseline model that included traditional clinical vascular risk factors resulted in a better model fit than traditional risk factors alone as indicated by goodnessof-fit statistics. Conclusions: In a multiethnic cohort of patients without cerebrovascular disease at baseline, we found that $\mathrm{CD}, \mathrm{CS}$, and IL-6 helped predict the occurrence of primary ischemic stroke. Future research could evaluate if these basic ultrasound and serum measurements have implications for primary prevention efforts or clinical trial inclusion criteria.

(c) 2021 The Author(s)

Published by S. Karger AG, Basel

\section{Introduction}

Because of the high morbidity and mortality from stroke and cerebrovascular disease, identifying those who may be at highest risk is of great public health importance. In order to identify those who may benefit from more aggressive risk reduction, clinicians often rely on risk pre- 
diction scores, such as the Framingham risk score $[1,2]$. Many of these methods of risk scoring rely solely on clinical factors, without utilizing relevant early imaging or laboratory findings that may point to intermediate vascular phenotypes such as atherosclerotic disease $[3,4]$.

Many carotid imaging findings are associated with increased stroke risk beyond well-established carotid stenosis, including increased carotid intima-media thickness (CIMT), carotid artery stiffness, and certain carotid plaque features such as intraplaque hemorrhage [5-8]. Of these, only later-stage markers of disease such as severe carotid stenosis currently drive changes in clinical management based on NASCET, CREST, and other trials [912]. Despite the established association of early sonographic imaging features with future cerebrovascular disease, there is conflicting data regarding the usefulness of earlier-stage markers of disease such as CIMT [13-15] to traditional risk prediction models $[16,17]$. In addition to imaging findings, there is compelling evidence that some markers of systemic inflammation are associated with stroke [18-20]. Prior studies have shown that serum inflammatory markers, including interleukin-6 (IL-6) and C-reactive protein (CRP), may improve cerebrovascular risk prediction and prognostication of stroke outcomes [21-23]. Since early imaging and laboratory markers are not routinely included in stroke risk prediction scores, we sought to evaluate whether including them would improve future stroke risk prediction. In a secondary analysis of the Multi-Ethnic Study of Atherosclerosis (MESA), we investigated whether findings on the baseline carotid ultrasound and serum inflammatory markers improved ischemic stroke risk prediction.

\section{Materials and Methods}

\section{Subjects}

MESA is a prospective epidemiologic study designed to identify clinical factors which may predict the development of ischemic symptoms in previously asymptomatic individuals [24]. With a local Institutional Review Board waiver, we obtained the MESA dataset from the NHLBI Biologic Specimen and Data Repository Information Coordinating Center. In brief, MESA participants included 6,814 men and women aged 45-84 years at baseline who were free of cerebrovascular disease, defined as no history of physician-diagnosis of a cerebrovascular event (ischemic or hemorrhagic stroke or TIA). The participants are from 4 racial/ethnic groups (White, Black, Hispanic, and Asian) and from households in 6 US population centers (Baltimore, MD; Chicago, IL; Forsyth County, NC; Los Angeles, CA; New York, NY; and St Paul, MN).

The dates for examinations in the MESA study were as follows: examination 1 between July 2000 and August 2002, exam- ination 2 from September 2002 to February 2004, examination 3 from March 2004 to September 2005, examination 4 from September 2005 to May 2007, and examination 5 from April 2010 to December 2011 [24-26]. Each participating site had approval from their respective Institutional Review Boards and all participants provided informed consent. Inclusion criteria were participants who had a carotid ultrasound examination and serum laboratory markers of inflammation at the baseline study visit.

\section{Ultrasound Examination}

Details of how carotid artery ultrasounds were obtained have been previously published [27]. Carotid stenosis was identified on Doppler ultrasound using peak systolic velocities in the internal carotid artery (ICA) and common carotid artery (CCA) by MESA investigators [24]. In an effort to include earlier stages of carotid stenosis, we created a binary variable with $0-24 \%$ stenosis and $>25 \%$ stenosis [28]. The intima-media thickness (IMT) of both the CCAs and ICAs were measured and reported in millimeters. Blinded replicate scans were performed on a subset of participants with intraclass correlation coefficients of 0.92 for CCA IMT and 0.88 for ICA IMT [27]. Interreader reproducibility was good with intraclass correlation coefficients of 0.81 for CCA IMT and 0.88 for ICA IMT [27]. Carotid plaque was defined as a discrete, focal wall thickening $\geq 1.5 \mathrm{~cm}$ or focal thickening at least $50 \%$ greater than the surrounding intima media (see online suppl. materials; see www.karger.com/doi/10.1159/000514373 for all online suppl. material) [29].

\section{Arterial Stiffness Measures}

All carotid stiffness measures were recorded with a B-mode ultrasound in the distal CCA using a Logiq 700 ultrasound machine (GE Medical Systems, Milwaukee, WI, USA) by the MESA investigators [30]. Briefly, carotid distensibility was defined as a ratio of the relative change in the cross-sectional area of the CCA over the cardiac cycle by the pulse pressure at the brachial artery. Peterson's elastic modulus was calculated as pulse pressure divided by the relative change in diameter of the carotid artery between the systole and diastole. Young's elastic modulus is a similar calculation of distensibility, but accounts for wall thickness by including a wall thickness term. It was calculated by dividing Peterson's elastic modulus by the wall thickness of the aorta. All of these indices are calculations of arterial stiffness, with lower values indicating a greater degree of arterial stiffness. The previously reported intraobserver and interobserver class correlation coefficient was 0.71 and 0.85 for carotid distensibility, respectively, and 0.69 and 0.84 for Young's modulus, respectively [30].

Total brachial artery reactivity, or dilatation of the brachial artery after the release of an occlusive blood pressure cuff, was also measured with a B-mode ultrasound using a Logiq-700 [31]. Intraobserver correlation coefficients for this method ranged from 0.50 to 0.90 [31]. Large and small artery elasticity, indicative of arterial stiffness, were measured noninvasively (see online suppl. materials for details) [32].

Measurement of Laboratory Biomarkers of Inflammation

We analyzed inflammatory markers of fibrinogen, CRP, plasmin-antiplasmin complex, factor VIII, D-dimer, and IL-6 measured at baseline for their association with future ischemic stroke (see online suppl. materials).
Cerebrovasc Dis Extra 2021;11:37-43 DOI: $10.1159 / 000514373$
Baradaran et al. 
Table 1. Baseline demographics shown for the full cohort and those with versus without ischemic stroke during follow-up $(n=5,918$, except where noted otherwise)

\begin{tabular}{|c|c|c|c|c|}
\hline & $\begin{array}{l}\text { Full cohort } \\
(n=5,918)\end{array}$ & $\begin{array}{l}\text { No ischemic } \\
\text { stroke }(n=5,813)\end{array}$ & $\begin{array}{l}\text { Ischemic } \\
\text { stroke }(n=105)\end{array}$ & $p$ value \\
\hline Age, years & $62.0(10.3)$ & $61.9(10.2)$ & $68.1(9.9)$ & $<0.001$ \\
\hline White & $2,264,38.3$ & $2,225,38.3$ & $39,37.1$ & 0.143 \\
\hline Black & $1,563,26.4$ & $1,530,26.3$ & $33,31.4$ & 0.239 \\
\hline Hispanic ethnicity & $1,349,23.2$ & $1,322,23.1$ & $27,26.5$ & 0.428 \\
\hline Systolic blood pressure & $126.4(21.4)$ & $126.1(21.3)$ & $141.8(22.2)$ & $<0.001$ \\
\hline Diastolic blood pressure & $72.0(10.3)$ & $71.9(10.3)$ & $76.3(9.9)$ & $<0.001$ \\
\hline Diabetes & $654,11.1$ & $627,10.8$ & $27,25.7$ & $<0.001$ \\
\hline Atrial fibrillation & $1,0.02$ & $1,0.02$ & 0,0 & 0.893 \\
\hline Weight & $172.7(37.6)$ & $172.6(37.6)$ & $178.0(36.8)$ & 0.142 \\
\hline Body mass index & $28.2(5.3)$ & $28.2(5.3)$ & $29.1(4.9)$ & 0.101 \\
\hline Intentional exercise (MET), min/week & $1,553.0(2,345.4)$ & $1,557.4(2,356.3)$ & $1,308.3(1,608.8)$ & 0.283 \\
\hline Family history of heart attack $(n=5,550)$ & $2,355,42.4$ & $2,307,42.3$ & $48,48.0$ & 0.256 \\
\hline Completed high school or less education $(n=5,904)$ & $2,148,36.4$ & $2,102,36.2$ & $46,44.2$ & 0.093 \\
\hline Current smoker & $745,12.6$ & $729,12.5$ & $16,15.2$ & 0.409 \\
\hline Current alcohol use $(n=4,744)$ & $3,272,69.0$ & $3,218,69.0$ & $54,67.5$ & 0.774 \\
\hline Left ventricular hypertrophy on electrocardiogram $(n=5,883)$ & $58,1.0$ & $56,1.0$ & $2,1.9$ & 0.336 \\
\hline Aspirin use $(n=5,662)$ & $1,111,19.6$ & $1,083,19.5$ & $28,29.5$ & 0.015 \\
\hline
\end{tabular}

Binary variables are presented as $n, \%$ and interval variables as the mean (SD). $p$ values are shown for the stroke vs. no stroke group and calculated with the Student $t$ test for interval variables and the $\chi^{2}$ test for binary variables. MET, metabolic equivalent.

Cerebrovascular Disease Follow-Up

The process for adjudicating cerebrovascular events in MESA has been previously published [24]. Ischemic strokes, TIAs, and hemorrhagic strokes were adjudicated by a MESA committee including neurologists and physician epidemiologists using data from a combination of medical records and in-person interviews with participants and family members of participants. Ischemic strokes were defined as fatal or nonfatal strokes due to ischemic brain infarction.

\section{Statistical Analysis}

We fit Cox proportional hazard models to ischemic stroke. Because there were complex interactions between covariates, we used least absolute shrinkage and selection operator (LASSO) regression analysis to select variables for the baseline model, which included 4 decades of patient age (44-54 vs. 55-64 vs. $65-74$ vs. $75-$ 84 years), 3 ordinal categories of total cholesterol (65-199 vs. 200239 vs. $\geq 240 \mathrm{mg} / \mathrm{dL}$ ), and binary variables (yes/no) of baseline hypertension, diabetes, current and former smoking, and baseline systolic blood pressure $\geq 160 \mathrm{~mm} \mathrm{Hg}$. We then calculated a likelihood ratio test and Akaike's information criterion to compare a baseline model for predicting ischemic stroke using standard clinical predictors including age, systolic blood pressure, cholesterol, diabetes, current smoking, and hypertension to a new model which added the predictor variables that remained significant from our regression analyses. For the new prediction model, we evaluated models using interactive variable selection and cutpoint determination and backward stepping with an $\alpha$-error criterion of 0.05 . We also calculated the net reclassification improvement (NRI) and integrated discrimination improvement (IDI) before and after adding the new markers in the model. The NRI reflects the sum of the percent improvement for events and non-events between the new and baseline model while the IDI evaluates the ability of additional biomarkers to predict an outcome of interest by estimating the percent change in prediction of the new model compared to the baseline model.

\section{Results}

After excluding 896 patients due to incomplete imaging and serum inflammatory marker data, we included 5,918 participants with an average age of 62.0 years at baseline (52.2\% female; Table 1$)$. With a mean follow-up 
Table 2. The baseline multivariate Cox proportional hazards model fit to the outcome of ischemic stroke

\begin{tabular}{lllr}
\hline Variable & Hazard ratio $^{1}$ & $95 \%$ CI & $p$ value \\
\hline Age (years) & 1.60 & $1.30-1.98$ & $<0.001$ \\
Hypertension & 2.52 & $1.56-4.08$ & $<0.001$ \\
Diabetes & 2.30 & $1.48-3.60$ & $<0.001$ \\
Total cholesterol & 1.40 & $1.07-1.84$ & 0.014 \\
Current smoking & 1.99 & $1.15-3.45$ & 0.014 \\
Systolic blood pressure & 2.03 & $1.24-3.32$ & 0.005 \\
\hline
\end{tabular}

${ }^{1}$ Adjusted for baseline age, diabetes, hypertension, total cholesterol, smoking, and systolic blood pressure $\geq 160 \mathrm{~mm} \mathrm{Hg}$.

time of 7.7 years ( 1.9 years), the subjects had a total of 105 ischemic strokes at a mean of 4.1 years of follow-up. We found that the ultrasound measures of carotid distensibility and carotid stenosis, and the laboratory marker of IL-6 were significantly associated with incident ischemic stroke when added to the baseline model with patient age, hypertension, diabetes, total cholesterol, smoking, and blood pressure (Table 2-4). The other evaluated imaging and laboratory values, including data on carotid plaque, were not significantly associated with incident ischemic stroke (Table 3, 4).

For ease of use in future risk prediction scores, we divided carotid distensibility and IL-6 into tertiles and then compared the baseline model to a new model with the addition of tertiles of carotid distensibility, IL-6, and categories of carotid stenosis. Goodness-of-fit of the baseline and new model was assessed by evaluating results from a likelihood ratio test and Akaike information criterion (AIC) of the 2 models. The likelihood ratio test resulted in a significant test statistic, suggesting that the new model provides a better fit to the data $(p<0.001)$. To further strengthen this claim, a decrease in AIC was observed from the baseline to model $(1,720.0$ to $1,703.2)$, indicating a better fit of the new model to the dataset. In addition, the new model that includes tertiles of carotid distensibility, IL-6, and categories of carotid stenosis shows better predictive capability than the baseline model based on concordance statistics ( 0.78 compared to 0.76 , respectively). We found positive values for both NRI and IDI with an NRI estimate of $0.198(p<0.001$, 95\% CI $0.084-0.338)$ and an IDI estimate of $0.012(p<$ $0.001,95 \%$ CI $0.002-0.065)$, indicating the addition of new biomarkers were beneficial in improving performance of the model.

\section{Discussion}

In a secondary analysis of a multiethnic cohort free of cerebrovascular disease at baseline, we found that carotid distensibility and $>25 \%$ carotid stenosis on ultrasound and a serum marker of inflammation predicted the occurrence of ischemic stroke over an average of an almost 8 -year period. Furthermore, we found that the addition of these basic sonographic parameters and serum measurements to a baseline ischemic stroke prediction model improved the model's ischemic risk prediction. While many traditional risk prediction scoring systems assess future risk for both hemorrhagic and ischemic strokes, we focused on the future prediction of only ischemic strokes because there are risk factors unique to ischemic strokes, including large artery atherosclerosis. We found that with the addition of some imaging and serum markers not usually included in many standard risk prediction modeling techniques, we were able to significantly improve ischemic stroke risk prediction.

Imaging markers such as those seen on carotid ultrasound can more directly visualize subclinical atherosclerosis and be helpful in improving stroke risk prediction. Though CIMT is strongly associated with future cerebrovascular ischemia, other studies, including a large metaanalysis, have found mixed utility of the addition of CIMT to standard stroke risk calculators [14,33]. Similarly, our analysis did not find the addition of measures of CIMT to be significant in improving stroke risk prediction. However, we found that carotid distensibility was significantly associated with incident stroke. Other studies have shown only marginal benefit in improving risk prediction models using arterial stiffness measurements [17]. Having increased arterial stiffness is thought to affect the pulsatility of small cerebral vessels and is associated with chronic microvascular changes and cerebral microbleeds [34]. Differences in utility of arterial stiffness measurements in the prediction of future stroke may be related to technique in measurement, for example more direct carotid stiffness measures, such as what was used in our cohort, may be more predictive compared to less direct assessment of arterial stiffness using peripheral brachial measurements. Also, decreased distensibility may be a marker of endothelial dysfunction, which can impair nitric oxide production and vascular reactivity leading to plaque production $[35,36]$.

Since carotid atherosclerotic disease is a direct cause of large artery atherosclerotic stroke, we expected to see increased risk of ischemic stroke in participants with carotid atherosclerotic plaque and stenosis. Other studies
Baradaran et al. 
Table 3. Effect of adding individual ultrasound variables to the baseline Cox proportional hazards model

\begin{tabular}{llll}
\hline Ultrasound variable & Hazard ratio $^{1}$ & 95\% CI & $p$ value \\
\hline Total brachial reactivity (\%) & 0.98 & $0.95-1.01$ & 0.206 \\
Large artery elasticity index & 0.99 & $0.94-1.03$ & 0.463 \\
Small artery elasticity index & 0.91 & $0.82-1.02$ & 0.101 \\
Total vascular impedance & 1.00 & $1.00-1.00$ & 0.745 \\
Carotid distensibility coefficient (×100) & 0.05 & $0.00-0.75$ & 0.030 \\
Carotid Youngs modulus & 1.00 & $1.00-1.00$ & 0.752 \\
Common CIMT (mm) & 0.92 & $0.33-2.53$ & 0.871 \\
Internal CIMT (mm) & 1.17 & $0.90-1.53$ & 0.244 \\
Z-score for maximum IMT & 1.07 & $1.21-3.27$ & 0.463 \\
Maximum carotid stenosis (0-24 vs. >25\%) & 1.98 & $1.29-3.04$ & 0.002 \\
Carotid plaque surface irregularity & 0.97 & $0.90-1.04$ & 0.353 \\
Carotid plaque echotexture & 1.12 & $0.96-1.30$ & 0.146 \\
\hline
\end{tabular}

Italicized variables are statistically significant. CIMT, carotid intima-media thickness.

${ }^{1}$ Adjusted for baseline age, diabetes, hypertension, total cholesterol, smoking, and systolic blood pressure $\geq 160 \mathrm{~mm} \mathrm{Hg}$.

Table 4. Effect of adding individual serum inflammatory marker variables to the baseline Cox proportional hazards model

\begin{tabular}{llcc}
\hline Serum inflammatory marker & Hazard ratio $^{1}$ & $95 \%$ CI & $p$ value \\
\hline CRP & 1.01 & $0.98-1.04$ & 0.433 \\
Fibrinogen antigen $(\mathrm{mg} / \mathrm{dL})$ & 1.00 & $1.00-1.00$ & 0.192 \\
Plasmin-antiplasmin complex $(\mathrm{nM})$ & 1.03 & $0.97-1.10$ & 0.324 \\
D-Dimer $(\mu \mathrm{g} / \mathrm{mL})$ & 0.95 & $0.71-1.26$ & 0.704 \\
Factor VIII $(\%)$ & 1.00 & $1.00-1.01$ & 0.608 \\
IL-6 $(\mathrm{pg} / \mathrm{mL})$ & 1.21 & $1.08-1.36$ & 0.001 \\
\hline
\end{tabular}

Italicized variables are statistically significant. CRP, C-reactive protein.

${ }^{1}$ Adjusted for baseline age, diabetes, hypertension, total cholesterol, smoking, and systolic blood pressure $\geq 160 \mathrm{~mm} \mathrm{Hg}$.

have shown improvements in stroke risk prediction with the addition of specific carotid plaque data $[16,17]$. Highrisk plaque features, such as echolucent plaque, are strongly associated with future cerebrovascular ischemia [6]. Though we showed that having some plaque leading to at least $25 \%$ stenosis is associated with incident ischemic stroke, our study did not demonstrate any improvements in stroke risk with the inclusion of specific carotid plaque characteristic data. Because of the low numbers of participants with high-risk plaque features, such as plaque surface irregularity, differences in stroke risk prediction were difficult to detect. Additionally, plaque volume was not accounted for in the analysis, which further limits the predictive ability of the included plaque data. This some- what limits extrapolation to patients with advanced atherosclerotic disease; however, future studies can be designed that target patients with these early imaging and inflammatory markers to determine if aggressive medical therapy can prevent vulnerable plaque features from developing.

Serum markers of systemic inflammation have also been used to improve stroke risk prediction, with CRP the most commonly cited [37]. These inflammatory markers are thought to play a key role in atherosclerotic plaque rupture, leading to thrombosis and cerebrovascular ischemia [38]. IL-6 specifically stimulates synthesis of acute phase proteins, such as CRP and fibrinogen, and also stimulates release of white blood cells. IL-6 also has strong 
evidence demonstrating its utility in predicting outcomes in the setting of acute stroke $[18,23,39]$ and is associated with cardiovascular morbidity [40], but has less evidence in predicting future cardiovascular events [38]. Our findings show that there may be a role for IL- 6 in predicting future stroke.

Our study has several limitations. First, we used data from the baseline visit for included participants so we did not account for changes in vascular risk factors over the entire study period. While this limits evaluation of changes to vascular risk factors over time, it is similar to how other risk prediction systems are structured and is an inherent limitation to risk prediction schemes. Another limitation is the general applicability of the findings to all populations. Since the included cohort were free of cerebrovascular disease at baseline, the risk prediction scoring may be different than those with more vascular risk factors at baseline. Additionally, atrial fibrillation did not contribute to any of the ischemic strokes included in our cohort, which limits the applicability of our findings to those who suffer from ischemic stroke secondary to cardioembolic causes. Furthermore, there are inherent limitations with the use of ultrasound in evaluating carotid plaque, including limited ability to evaluate specific plaque components, such as intraplaque hemorrhage. Another inherent limitation in the data is the lack of baseline brain imaging. Future studies including baseline brain MRIs to evaluate for markers of cerebral small vessel disease, including white matter hyperintensities, covert brain infarctions, and cerebral microbleeds, may allow for improved stroke risk prediction. Lastly, adding markers of carotid artery disease and serum inflammatory markers improved stroke risk prediction modestly, perhaps in part due to the relatively young and cardiovascular risk factor-free cohort. Future studies in cohorts with higher baseline risk will be helpful to confirm our findings.

We found that sonographic measures of atherosclerosis including carotid stenosis and carotid distensibility, and a serum inflammatory marker, IL-6, were significantly associated with increased risk of ischemic stroke in a population free from cerebrovascular disease. We found that including these markers in risk prediction scoring systems also significantly improved risk prediction. With further validation, adding these basic measurements to prediction models could improve primary prevention efforts with more focused primary prevention strategies and could be used to enroll patients in prospective clinical trials.

\section{Statement of Ethics}

This research complied with the guidelines for human studies and should include evidence that the research was conducted ethically in accordance with the World Medical Association Declaration of Helsinki. With a local Institutional Review Board waiver, we used de-identified data.

\section{Conflict of Interest Statement}

The authors have no conflicts of interest to declare.

\section{Funding Sources}

This work was supported by the Association of University Radiologists, GE Radiology Research Academic Fellowship and American Heart Association (grant No. 17SDG33460420).

\section{Author Contributions}

H.B. and A.D.H.: substantial contributions to the conception or design of the work; or the acquisition, analysis, or interpretation of data for the work; drafting the work or revising it critically for important intellectual content; final approval of the version to be published; and agreement to be accountable for all aspects of the work in ensuring that questions related to the accuracy or integrity of any part of the work are appropriately investigated and resolved. A.D., K.-H.W., N.S., J.S.M., M.A., and J.J.M.: substantial contributions to the conception or design of the work; revising the work critically for important intellectual content; final approval of the version to be published; and agreement to be accountable for all aspects of the work in ensuring that questions related to the accuracy or integrity of any part of the work are appropriately investigated and resolved.

References

1 Wilson PW, D'Agostino RB, Levy D, Belanger AM, Silbershatz H, Kannel WB. Prediction of coronary heart disease using risk factor categories. Circulation. 1998 May;97(18):1837-47.

2 D'Agostino RB, Wolf PA, Belanger AJ, Kannel WB. Stroke risk profile: adjustment for antihypertensive medication. The Framingham Study. Stroke. 1994 Jan;25(1):40-3.

3 Hemann BA, Bimson WF, Taylor AJ. The Framingham Risk Score: an appraisal of its benefits and limitations. Am Heart Hosp J. 2007;5(2):91-6.

4 Schlendorf KH, Nasir K, Blumenthal RS. Limitations of the Framingham risk score are now much clearer. Prev Med. 2009 Feb;48(2):115-6.

5 Romero JR, Beiser A, Seshadri S, Benjamin EJ, Polak JF, Vasan RS, et al. Carotid artery atherosclerosis, MRI indices of brain ischemia, aging, and cognitive impairment: the Framingham study. Stroke. 2009 May;40(5): 1590-6.
Baradaran et al. 
6 Gupta A, Kesavabhotla K, Baradaran H, Kamel H, Pandya A, Giambrone AE, et al. Plaque echolucency and stroke risk in asymptomatic carotid stenosis: a systematic review and meta-analysis. Stroke. 2015 Jan;46(1):91-7.

7 Gupta A, Baradaran H, Schweitzer AD, Kamel H, Pandya A, Delgado D, et al. Carotid plaque MRI and stroke risk: a systematic review and meta-analysis. Stroke. 2013 Nov; 44(11):3071-7.

8 Dijk JM, van der Graaf Y, Grobbee DE, Bots ML; SMART Study Group. Carotid Stiffness Indicates Risk of Ischemic Stroke and TIA in Patients With Internal Carotid Artery Stenosis. Stroke. 2004;35(10):2258-62.

9 Rothwell PM, Eliasziw M, Gutnikov SA, Fox AJ, Taylor DW, Mayberg MR, et al.; Carotid Endarterectomy Trialists' Collaboration. Analysis of pooled data from the randomised controlled trials of endarterectomy for symptomatic carotid stenosis. Lancet. 2003 Jan; 361(9352):107-16.

10 Barnett HJ, Taylor DW, Haynes RB, Sackett DL, Peerless SJ, Ferguson GG, et al.; North American Symptomatic Carotid Endarterectomy Trial Collaborators. Beneficial effect of carotid endarterectomy in symptomatic patients with high-grade carotid stenosis. N Engl J Med. 1991 Aug;325(7):445-53.

11 Group EC. Randomised trial of endarterectomy for recently symptomatic carotid stenosis: final results of the MRC European Carotid Surgery Trial (ECST). Lancet. 1998 May; 351(9113):1379-87.

12 Walker MD, Marler JR, Goldstein M, Grady PA, Toole JF, Baker WH, et al. Endarterectomy for asymptomatic carotid artery stenosis. Executive Committee for the Asymptomatic Carotid Atherosclerosis Study. JAMA. 1995 May;273(18):1421-8.

13 van den Oord SC, Sijbrands EJ, ten Kate GL, van Klaveren D, van Domburg RT, van der Steen AF, et al. Carotid intima-media thickness for cardiovascular risk assessment: systematic review and meta-analysis. Atherosclerosis. 2013 May;228(1):1-11.

14 Den Ruijter HM, Peters SA, Anderson TJ, Britton AR, Dekker JM, Eijkemans MJ, et al. Common carotid intima-media thickness measurements in cardiovascular risk prediction: a meta-analysis. JAMA. 2012 Aug; 308(8):796-803.

15 Nambi V, Chambless L, Folsom AR, He M, $\mathrm{Hu} \mathrm{Y}$, Mosley T, et al. Carotid intima-media thickness and presence or absence of plaque improves prediction of coronary heart disease risk: the ARIC (Atherosclerosis Risk In Communities) study. J Am Coll Cardiol. 2010 Apr 55(15):1600-7.

16 Naqvi TZ, Lee MS. Carotid intima-media thickness and plaque in cardiovascular risk assessment. JACC Cardiovasc Imaging. 2014 Oct;7(10):1025-38.

17 Störk S, van den Beld AW, von Schacky C, Angermann CE, Lamberts SW, Grobbee DE, et al. Carotid artery plaque burden, stiffness, and mortality risk in elderly men: a prospective, population-based cohort study. Circulation. 2004 Jul;110(3):344-8.

18 Tarkowski E, Rosengren L, Blomstrand C, Wikkelsö C, Jensen C, Ekholm S, et al. Early intrathecal production of interleukin- 6 predicts the size of brain lesion in stroke. Stroke. 1995 Aug;26(8):1393-8.

19 Kim JS, Yoon SS, Kim YH, Ryu JS. Serial measurement of interleukin-6, transforming growth factor- $\beta$, and $S-100$ protein in patients with acute stroke. Stroke. 1996 Sep;27(9): 1553-7.

20 Ferrarese C, Mascarucci P, Zoia C, Cavarretta R, Frigo M, Begni B, et al. Increased cytokine release from peripheral blood cells after acute stroke. J Cereb Blood Flow Metab. 1999 Sep; 19(9):1004-9.

21 Rallidis LS, Vikelis M, Panagiotakos DB, Rizos I, Zolindaki MG, Kaliva K, et al. Inflammatory markers and in-hospital mortality in acute ischaemic stroke. Atherosclerosis. 2006 Nov;189(1):193-7.

22 Elkind MS. Inflammation, atherosclerosis, and stroke. Neurologist. 2006 May;12(3): 140-8.

23 Whiteley W, Jackson C, Lewis S, Lowe G, Rumley A, Sandercock P, et al. Inflammatory markers and poor outcome after stroke: a prospective cohort study and systematic review of interleukin-6. PLoS Med. 2009 Sep; 6(9):e1000145.

24 Bild DE, Bluemke DA, Burke GL, Detrano R, Diez Roux AV, Folsom AR, et al. Multi-ethnic study of atherosclerosis: objectives and design. Am J Epidemiol. 2002 Nov 1;156(9): 871-81.

25 Blaha MJ, Cainzos-Achirica M, Greenland P, McEvoy JW, Blankstein R, Budoff MJ, et al. Role of coronary artery calcium score of zero and other negative risk markers for cardiovascular disease: the Multi-Ethnic Study of Atherosclerosis (MESA). Circulation. 2016 Mar; 133(9):849-58.

26 Olson JL, Bild DE, Kronmal RA. Burke GLJGh. Legacy of MESA. Glob Heart. 2016; 11(3):269-74

27 Polak JF, Szklo M, O’Leary DH. Carotid Intima-Media Thickness Score, Positive Coronary Artery Calcium Score, and Incident Coronary Heart Disease: The Multi-Ethnic Study of Atherosclerosis. J Am Heart Assoc. 2017 Jan;6(1):e004612.

28 Polak JF, Tracy R, Harrington A, Zavodni AE, O'Leary DH. Carotid artery plaque and progression of coronary artery calcium: the multi-ethnic study of atherosclerosis. J Am Soc Echocardiogr. 2013 May;26(5):548-55.

29 Tattersall MC, Gassett A, Korcarz CE, Gepner AD, Kaufman JD, Liu KJ, et al. Predictors of carotid thickness and plaque progression during a decade: the Multi-Ethnic Study of Atherosclerosis. Stroke. 2014 Nov;45(11):325762.

30 Blaha MJ, Budoff MJ, Rivera JJ, Katz R, O'Leary DH, Polak JF, et al. Relationship of carotid distensibility and thoracic aorta calcification: multi-ethnic study of atherosclerosis. Hypertension. 2009 Dec;54(6):1408-15.

31 Polak JF, Ouyang P, Vaidya D. Total brachial artery reactivity and first time incident coronary heart disease events in a longitudinal cohort study: the multi-ethnic study of atherosclerosis. PLoS One.2019 Apr;14(4):e0211726.

32 Finkelstein SM, Cohn JN. First- and third-order models for determining arterial compliance. J Hypertens Suppl. 1992 Aug;10(6):S11-4.

33 del Sol AI, Moons KG, Hollander M, Hofman A, Koudstaal PJ, Grobbee DE, et al. Is carotid intima-media thickness useful in cardiovascular disease risk assessment? The Rotterdam Study. Stroke. 2001 Jul;32(7):1532-8.

34 Poels MM, Zaccai K, Verwoert GC, Vernooij MW, Hofman A, van der Lugt A, et al. Arterial stiffness and cerebral small vessel disease: the Rotterdam Scan Study. Stroke. 2012 Oct; 43(10):2637-42.

35 Wilkinson IB, Qasem A, McEniery CM, Webb DJ, Avolio AP, Cockcroft JR. Nitric oxide regulates local arterial distensibility in vivo. Circulation. 2002 Jan;105(2):213-7.

36 Chlumský I, Charvát J. Endothelial dysfunction, distensibility and intima-media thickness and aetiology of stroke. J Int Med Res. 2005 Sep-Oct;33(5):555-61.

37 Di Napoli M, Papa F, Bocola V. C-reactive protein in ischemic stroke: an independent prognostic factor. Stroke. 2001 Apr;32(4): 917-24.

38 Herder C, Schöttker B, Rothenbacher D, Roden M, Kolb H, Müller H, et al. Interleukin- 6 in the prediction of primary cardiovascular events in diabetes patients: results from the ESTHER study. Atherosclerosis. 2011 May;216(1):244-7.

39 Bustamante A, Sobrino T, Giralt D, GarcíaBerrocoso T, Llombart V, Ugarriza I, et al. Prognostic value of blood interleukin- 6 in the prediction of functional outcome after stroke: a systematic review and meta-analysis. J Neuroimmunol. 2014 Sep;274(1-2):215-24.

40 Danesh J, Kaptoge S, Mann AG, Sarwar N, Wood A, Angleman SB, et al. Long-term interleukin-6 levels and subsequent risk of coronary heart disease: two new prospective studies and a systematic review. PLoS Med. 2008 Apr;5(4):e78.
Improved Stroke Risk Prediction with Ultrasound and Inflammation
Cerebrovasc Dis Extra 2021;11:37-43 DOI: $10.1159 / 000514373$ 